Supplementary Material for

\title{
Preparation of porous carbons from petroleum pitch and polyaniline by thermal treatment for methane storage
}

Navarro Quirant, P.; Cuadrado- Collados, C.; Romero-Anaya A.J.; Silvestre Albero, J.; Martinez Escandell, $\mathrm{M}^{*}$.

Laboratorio de Materiales Avanzados, Departamento de Química Inorgánica-Instituto Universitario de Materiales. Universidad de Alicante. Apartado 99, E-3080. Alicante, Spain 


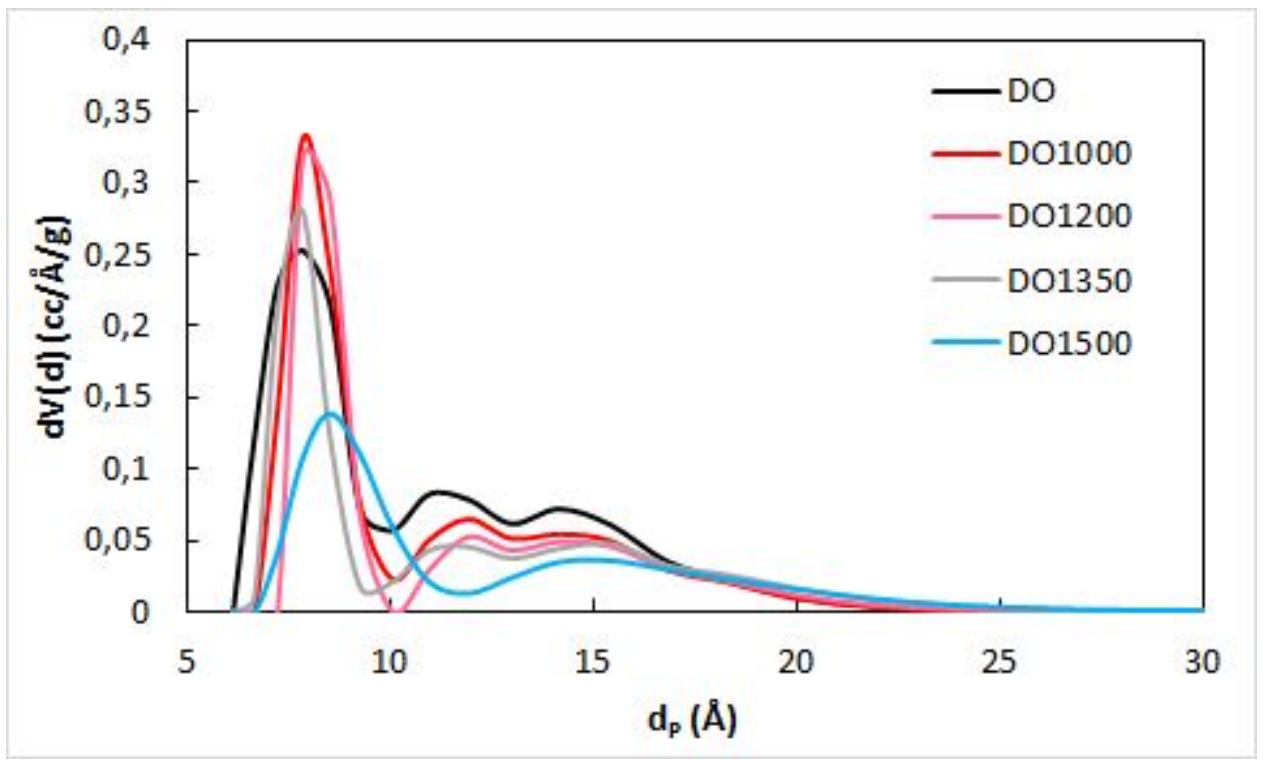

a)

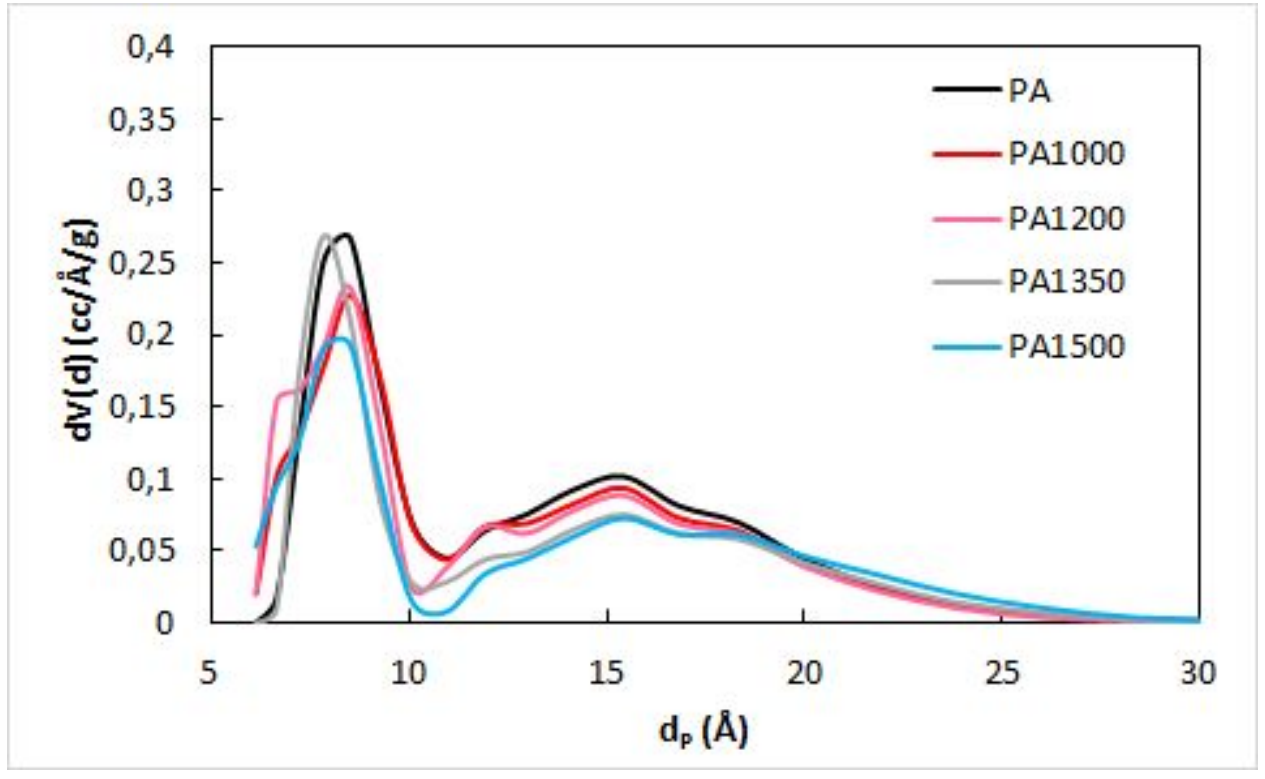

b)

Figure S1. Pore size distribution estimated from by N2 adsorption data after application of the QSDFT : a) DO series; b) PA series 


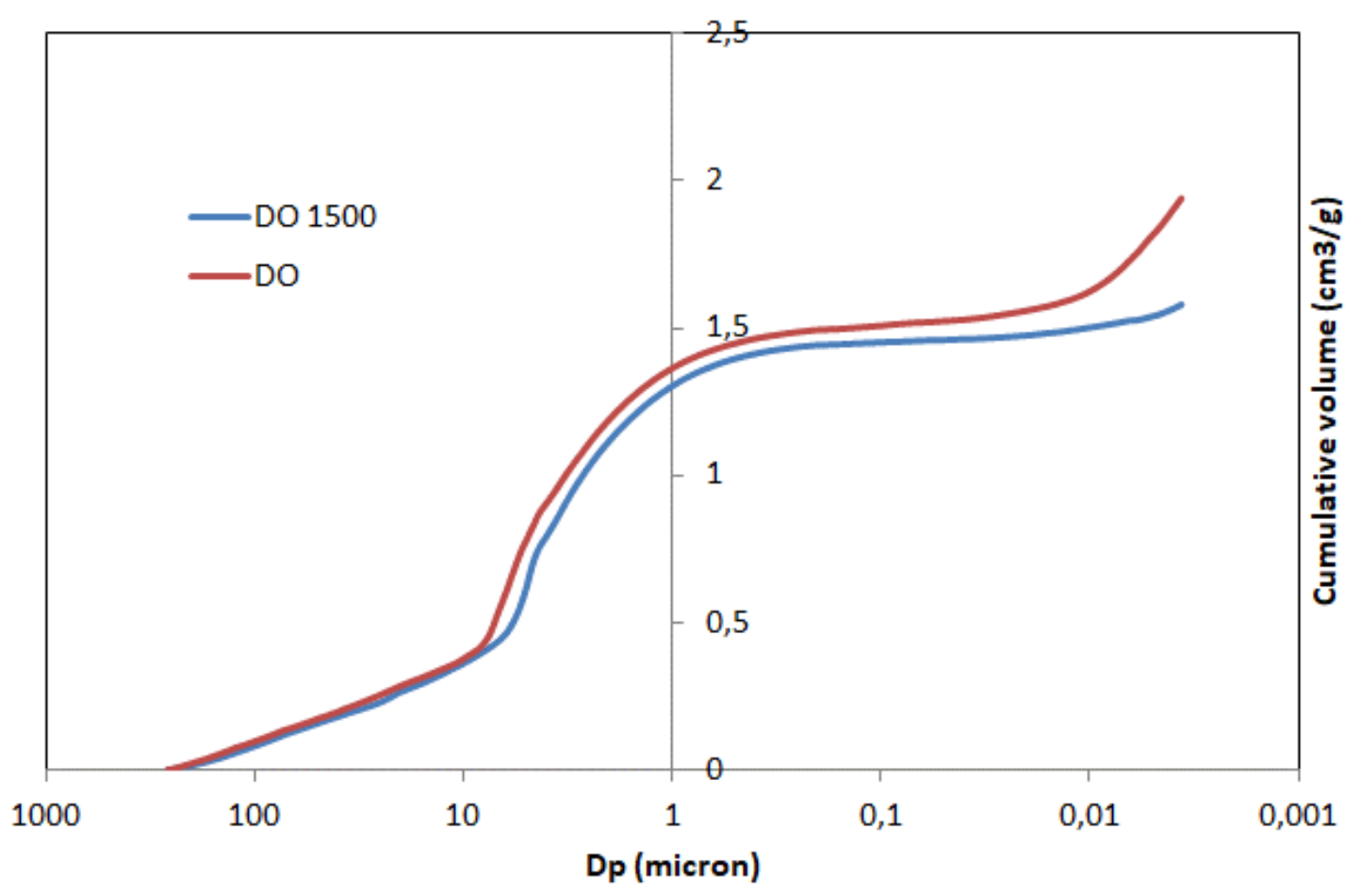

a)

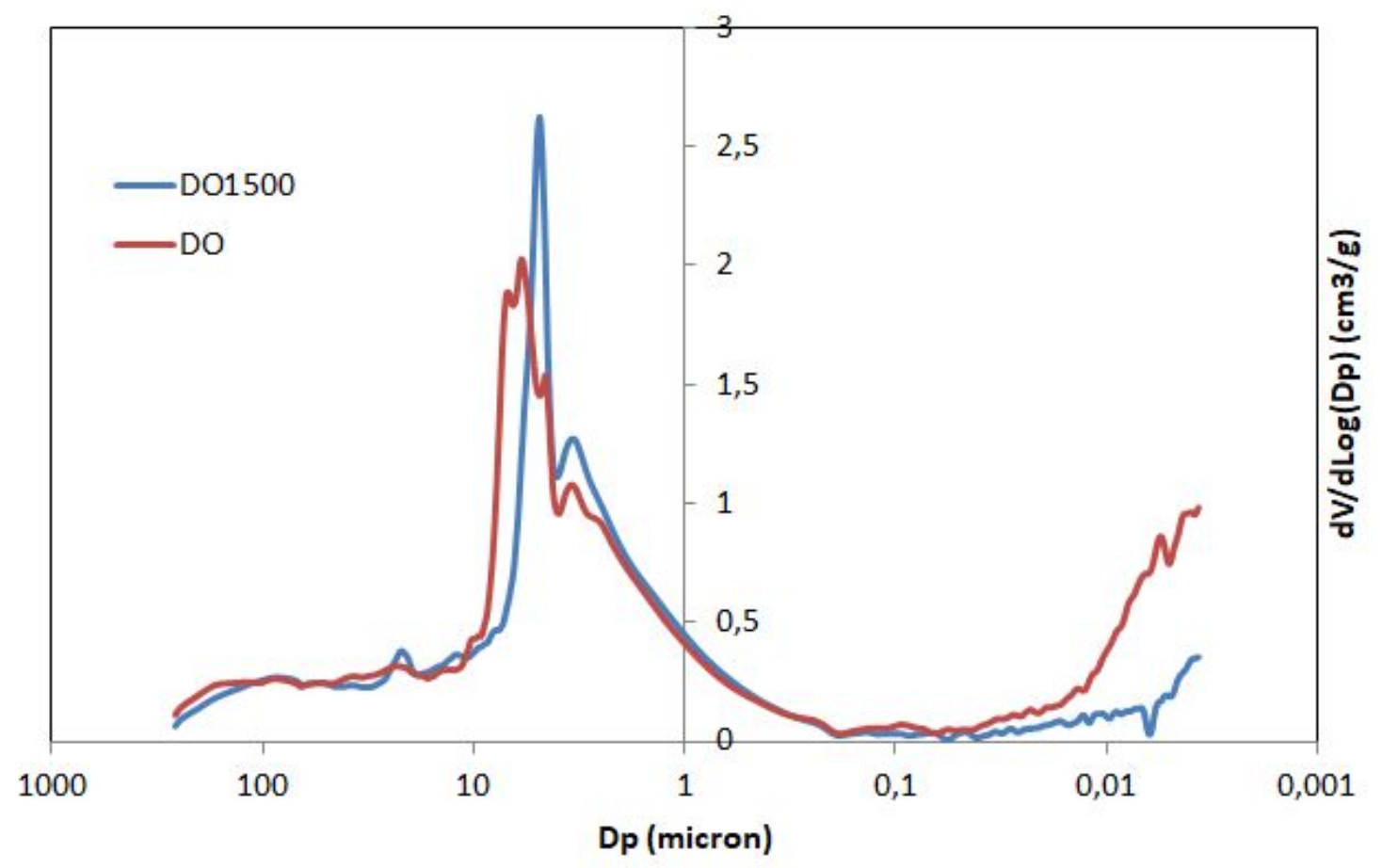

b)

Figure S2. Hg porosimetry of DO and DO100:a) cumulative volume vs pore diameter; b) pore size distribution (as $\mathrm{dV} / \mathrm{d} \log (\mathrm{Dp})$ ) vs. pore diameter. 


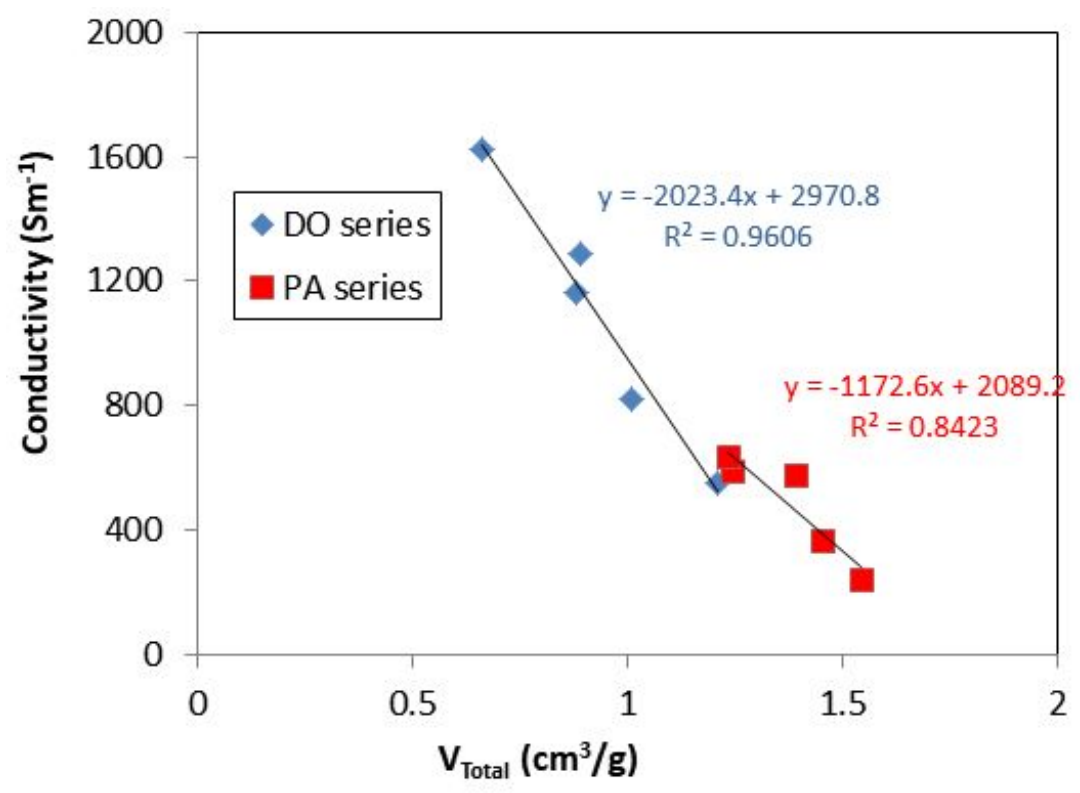

Figure S3. Electrical conductivity (S/m) vs Total pore volume for DO and PA Series

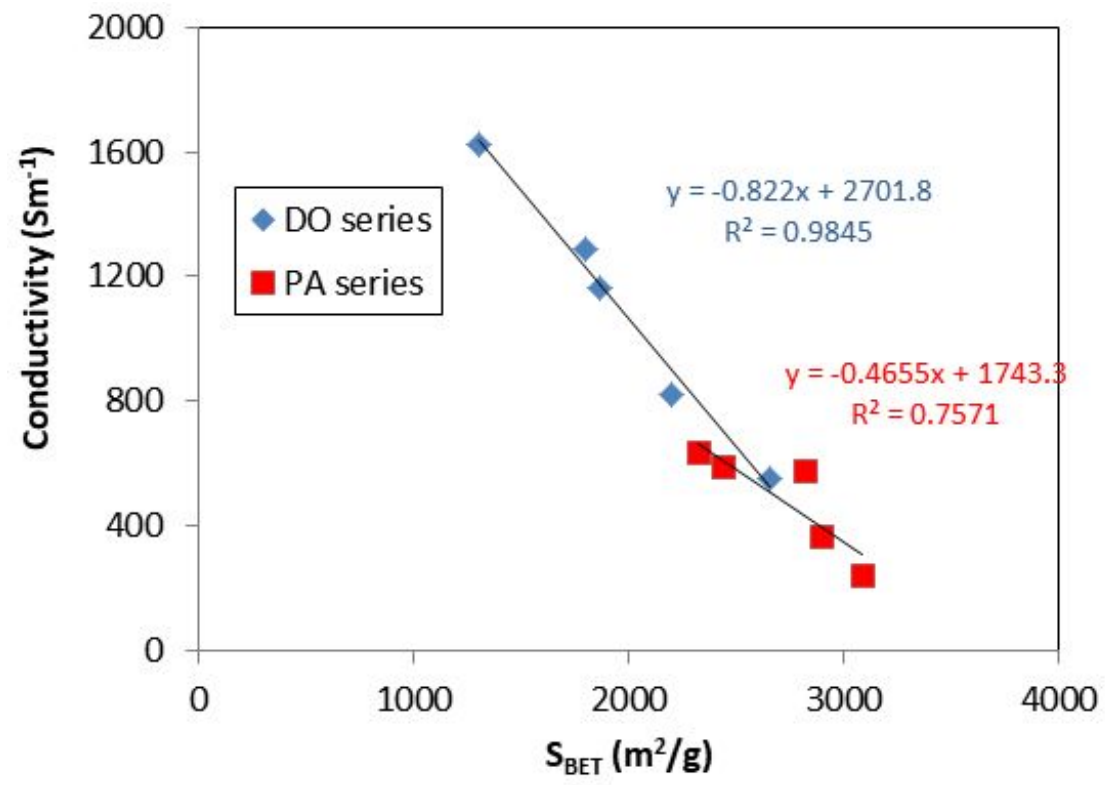

Figure S4. Electrical conductivity $(\mathrm{S} / \mathrm{m})$ vs BET surface area for DO and PA Series 


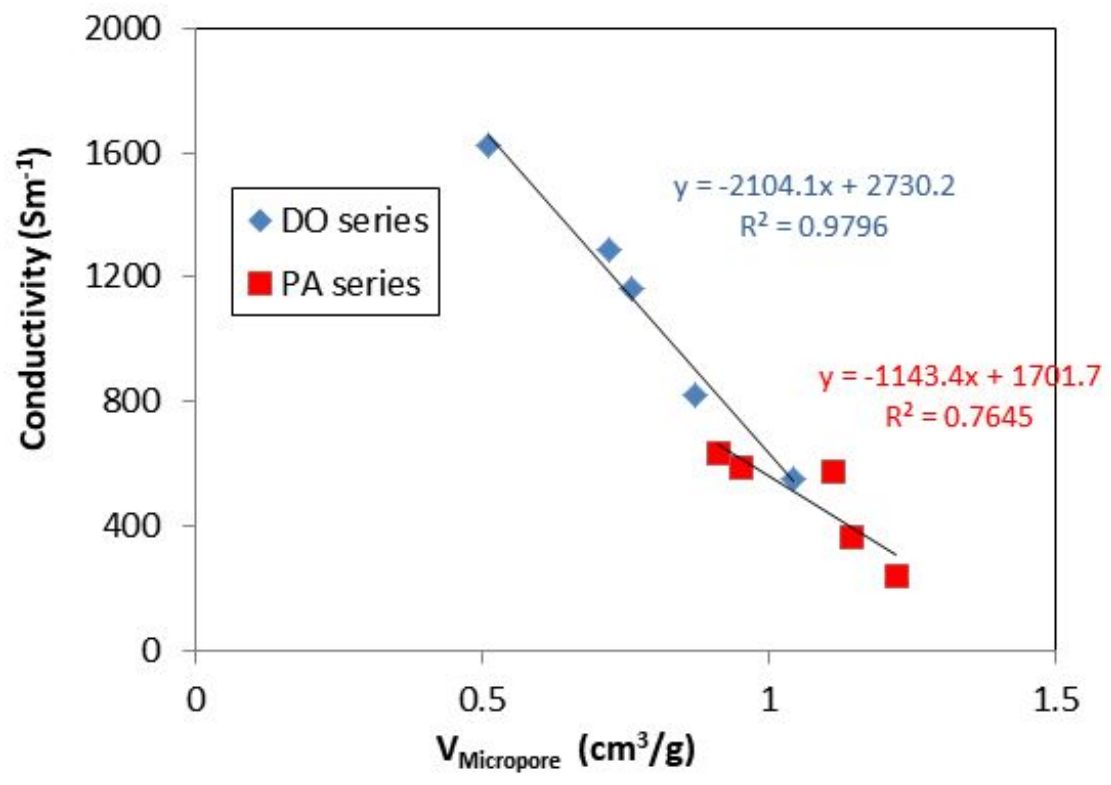

Figure S5 Electrical conductivity $(\mathrm{S} / \mathrm{m})$ vs micropore volume for DO and PA Series

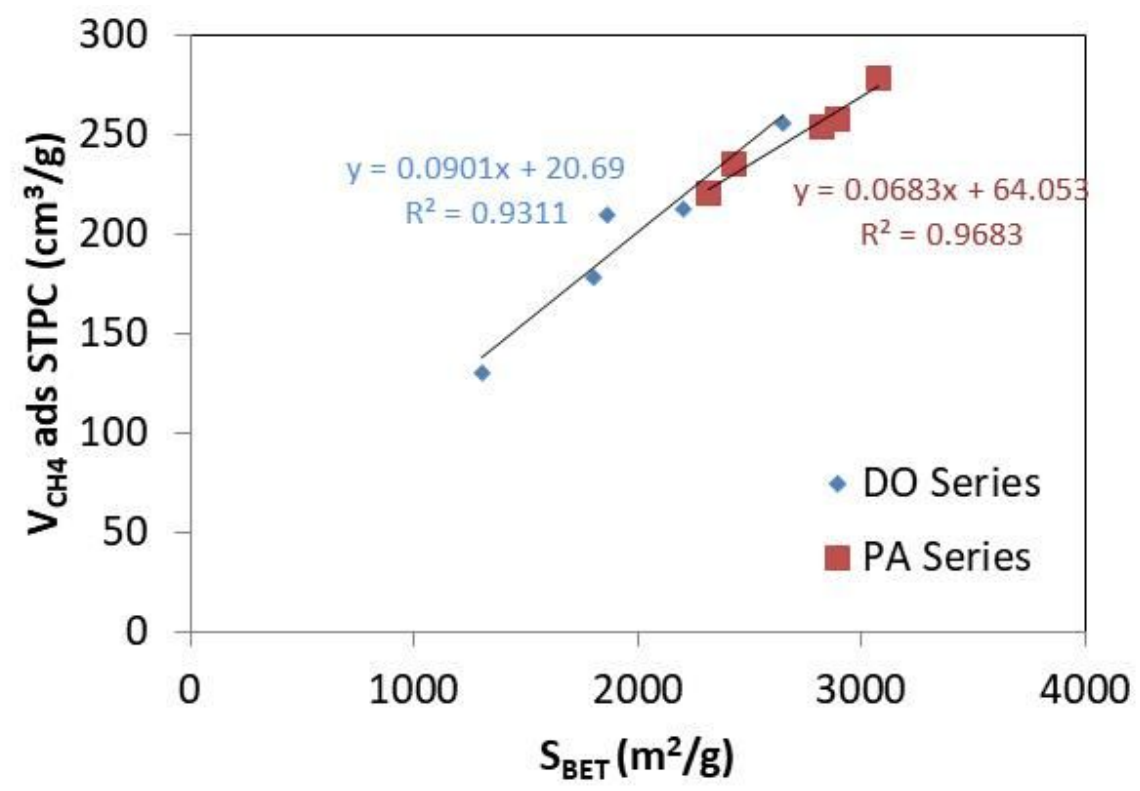

Figure S6. Methane adsorbed (V/g) vs BET surface area for DO and PA Series 


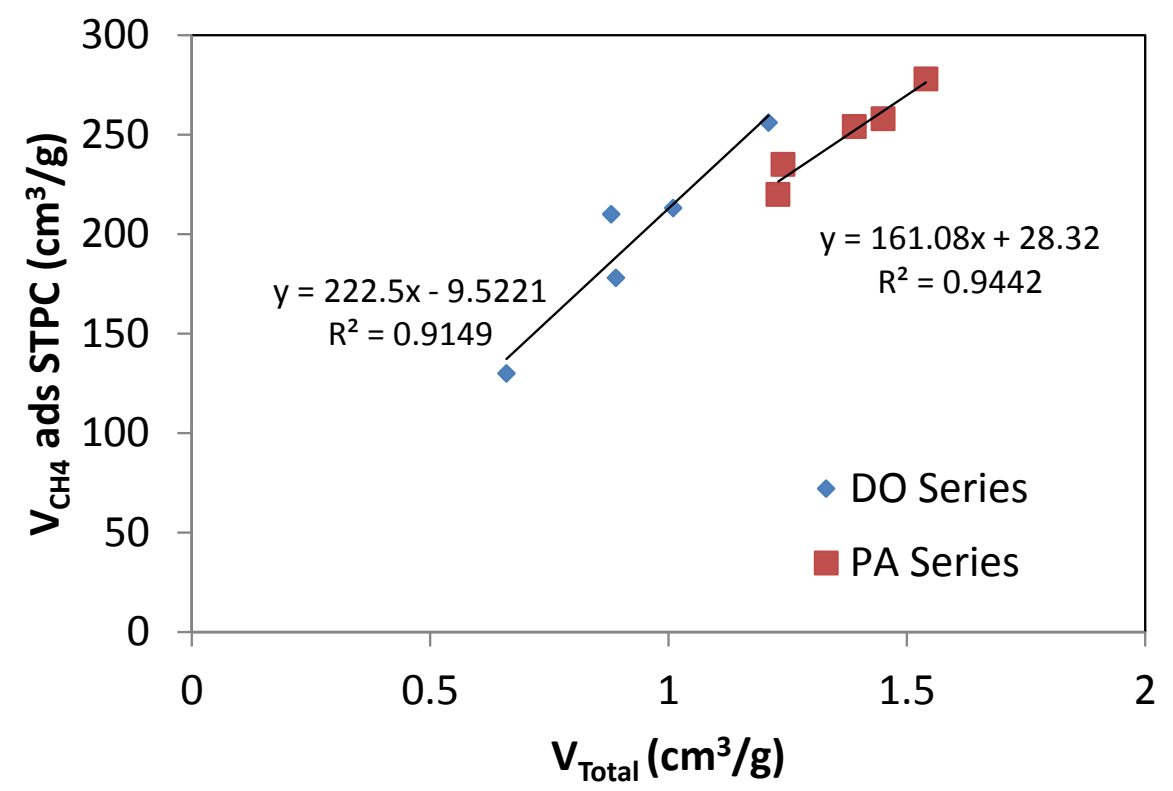

Figure S7. Methane adsorbed (V/g) vs Total Pore Volume for DO and PA Series

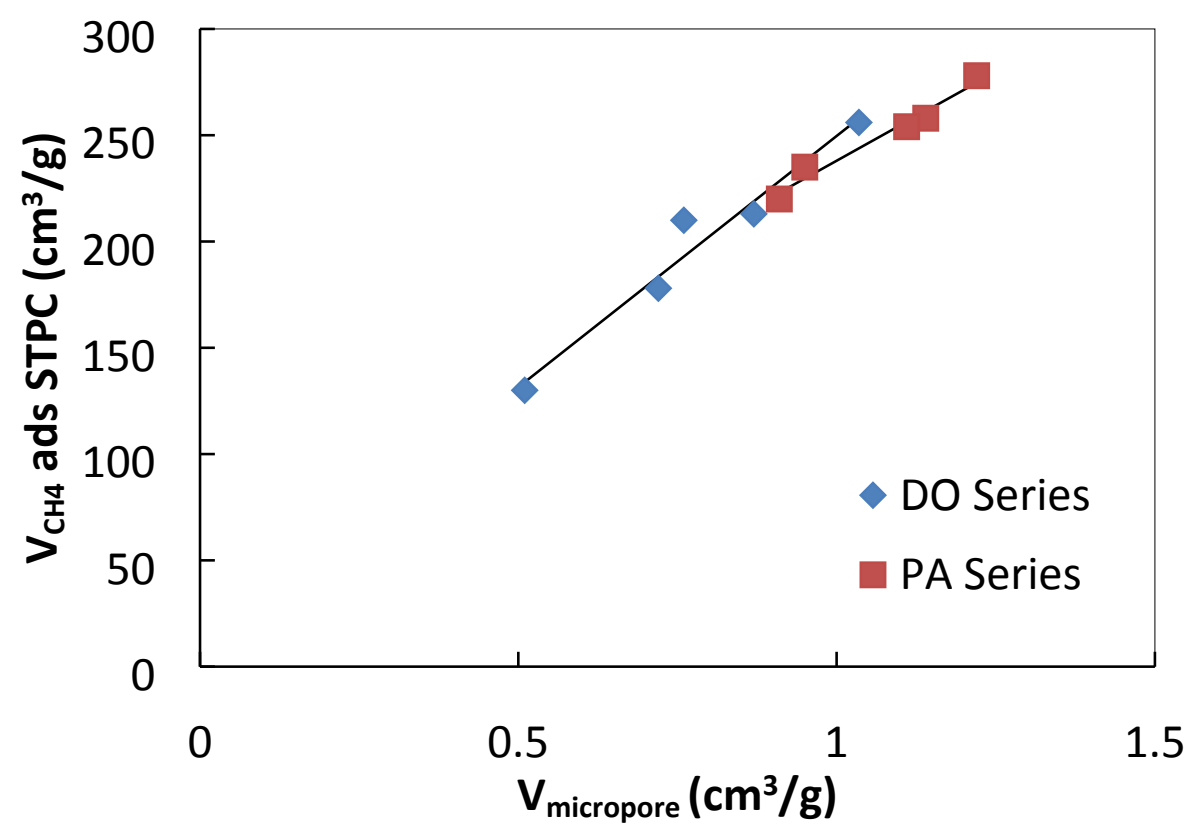

Figure S8. Methane adsorbed (V/g) vs Micropore volume for DO and PA Series 


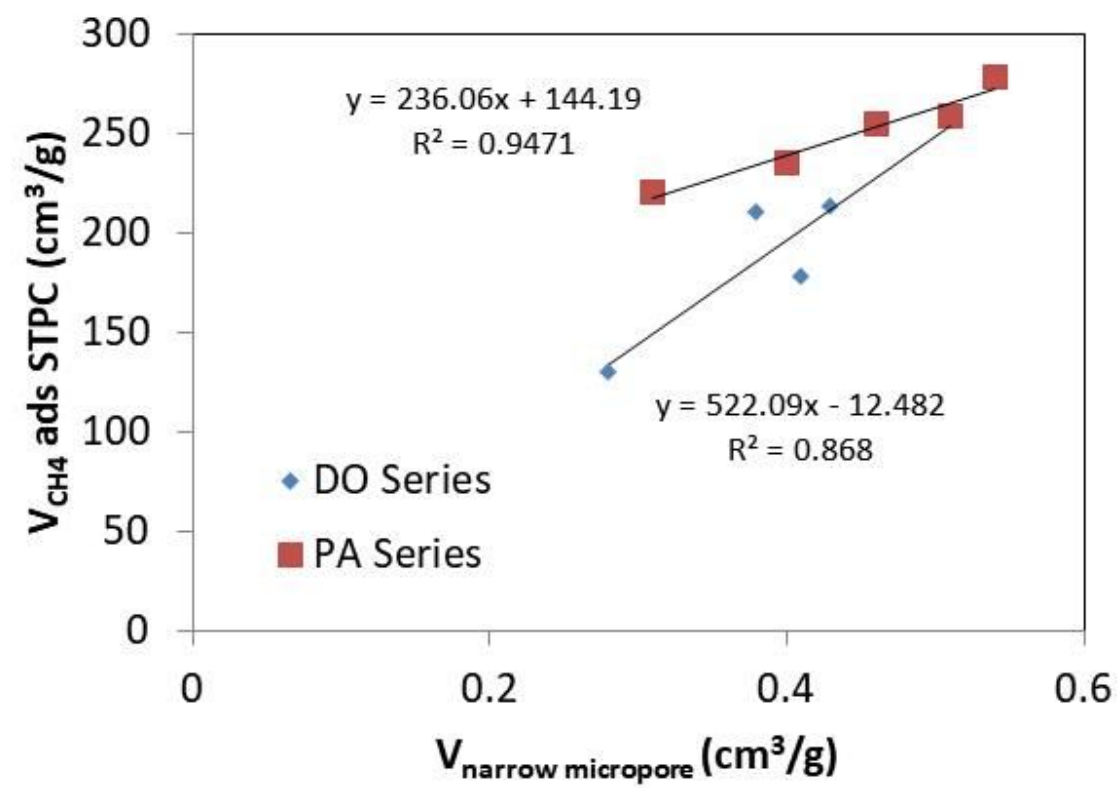

Figure S9. Methane adsorbed (V/g) vs narrow micropore volume for DO and PA Series 